\title{
A critical zone observatory dedicated to suspended sediment transport: the meso-scale Galabre catchment (southern French Alps)
}

\author{
Cédric Legout ${ }^{1}$, Guilhem Freche ${ }^{1}$, Romain Biron ${ }^{2}$, Michel Esteves ${ }^{2}$, Oldrich Navratil ${ }^{3}$, \\ Guillaume Nord ${ }^{1}$, Magdalena Uber $^{1}$, Thomas Grangeon ${ }^{4}$, Nico Hachgenei ${ }^{1}$, Brice \\ Boudevillain $^{1}$, Céline Voiron ${ }^{2}$, and Lorenzo Spadini ${ }^{1}$ \\ ${ }^{1}$ Université Grenoble Alpes \\ ${ }^{2}$ Institut de Recherche pour le Developpement IRD \\ ${ }^{3}$ Université Lumière Lyon 2 \\ ${ }^{4} \mathrm{BRGM}$
}

September 21, 2020

\begin{abstract}
The $20 \mathrm{~km}^{2}$ Galabre catchment belongs to the French network of critical zone observatories. It is representative of the sedimentary geology and meteorological forcing found in Mediterranean and mountainous areas. Due to the presence of highly erodible and sloping badlands of various lithologies, the site was instrumented in 2007 to understand the dynamics of suspended sediments (SS) in such areas. Two meteorological stations including measurements of air temperature, wind speed and direction, air moisture, rainfall intensity, raindrop size and velocity distribution are installed both in the upper and lower part of the catchment. At the catchment outlet, a gauging station records the water level, temperature and the turbidity (10 min. timestep). Water and sediment samples are collected automatically to estimate SS concentration-turbidity relationships, providing SS fluxes quantifications with known uncertainties. The sediment samples are further characterized by measuring their particle size distributions (PSD) and by applying a low-cost sediment fingerprinting approach using spectrocolorimetric tracers. Thus, the contributions of badlands on different lithologies to total SS flux are quantified at a high temporal resolution providing the opportunity to better analyze the links between meteorological forcing variability and watershed hydrosedimentary response. The set of measurements was extended to the dissolved phase in 2017. Both the river electrical conductivity and its major ion concentrations are measured each week and every three hours during storm events. This allows progress in understanding both the origin of the water during the events and the partitioning between particulate and dissolved fluxes in the critical zone.
\end{abstract}

\section{Hosted file}

Data_note_Galabre_observatory_Legout_main.pdf available at https://authorea.com/users/360629/ articles/482250-a-critical-zone-observatory-dedicated-to-suspended-sediment-transportthe-meso-scale-galabre-catchment-southern-french-alps 
(a)

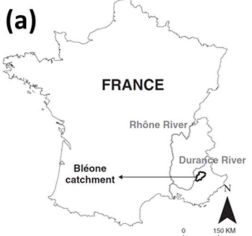

(b)

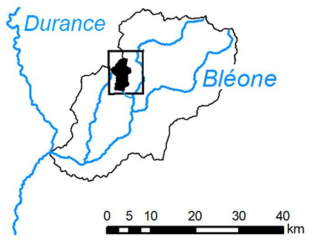

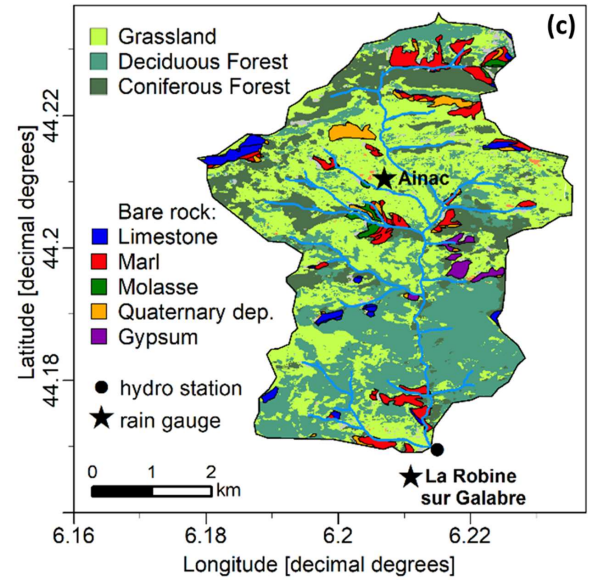

Figure 1 : (a) Location of the Bléone catchment including (b) the Galabre catchment. (c) Land cover map of the Galabre catchment adapted from CESBIO land cover data (Inglada et al., 2017). Badland areas were delineated in Legout et al. (2013) and classified according to their geology. 


\begin{tabular}{|c|c|c|c|c|c|}
\hline \multirow{6}{*}{ Meteorology } & Site name & Measurement & OSUG doi data access & BDOH data visualization & Period \\
\hline & $\begin{array}{l}\text { La Robine } \\
\text { sur Galabre }\end{array}$ & $\begin{array}{l}\text { Temperature, } \\
\text { moisture, wind speed } \\
\text { and direction, } \\
\text { precipitation }\end{array}$ & hittps://ddio.org/10.17778//DRAXXBLEONE_GAL_ROB_MEF_1420 & https://bdoh.irstea.ff/DRAIX/ROBINE & \\
\hline & & Drop size distribution & hittps://ddi.org/10.17178//DRAXXELEONE_GAL_ROB_OSD_1420 & & $14-20$ \\
\hline & Ainac, & $\begin{array}{l}\text { Temperature, } \\
\text { moisture, wind speed } \\
\text { and direction, } \\
\text { precipitation }\end{array}$ & https://doi.org/10.17178//DRAXXELEONE_GAL_AIN_MET_1920 & https://bdoh.irstea.fi/DRAIX/AINAC & $19-20$ \\
\hline & & Precipitation & 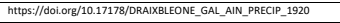 & hitps://bdoh.irstea.ff/DRAII/AINAC/PRCP & 08-19 \\
\hline & & Drop size distribution & 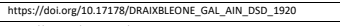 & & $19-20$ \\
\hline \multirow[t]{2}{*}{ Hydrology } & $\begin{array}{l}\text { La Robine } \\
\text { sur Galabre, }\end{array}$ & Liquid discharge & https:/ddo.org/10.17178/DRAAXBLEONE_GAL_ROB__IICH_0719 & https://bdoh.irstea.fr/DRAI//GALABBE/DEB & 07-19 \\
\hline & & Water temperature & hittps://ddio.org/10.17178/DRAIXELEONE_GAL_ROB_TEMP__1519 & $\begin{array}{l}\text { https:///doho.irstea.ff/DRAII/GALABRE/TEMPE } \\
\end{array}$ & $15-19$ \\
\hline \multirow[t]{3}{*}{$\begin{array}{l}\text { Particulate } \\
\text { matter }\end{array}$} & $\begin{array}{l}\text { La Robine } \\
\text { sur Galabre }\end{array}$ & $\begin{array}{l}\text { Suspended sediment } \\
\text { concentration }\end{array}$ & hhtps://ddio.org/10.17778//RAAXXELEONE_GAL_ROB__SS__0719 & hittps://bdoh.irstea.ff/DRAII/GALABRE/CMES & 07-19 \\
\hline & & $\begin{array}{l}\text { Particle size } \\
\text { distribution }\end{array}$ & hitps://ddi.org/10.17178/DRAIXBELEONE_GAL_ROB_EEDPSD_0809 & & $08-09$ \\
\hline & & $\begin{array}{l}\text { Sediment } \\
\text { fingerprinting }\end{array}$ & https://doi.org/10.17178/ORAIXBLEONE_GAL_ROB__EEDSOURCE_0719 & & 07-19 \\
\hline \multirow[t]{2}{*}{$\begin{array}{l}\begin{array}{l}\text { Dissolved } \\
\text { matter }\end{array} \\
\end{array}$} & $\begin{array}{l}\text { La Robine } \\
\text { sur Galabre }\end{array}$ & $\begin{array}{l}\text { Water electrical } \\
\text { conductivity }\end{array}$ & hhtps://ddio.org/10.17178/DRAAXELEONE_GAL_ROB_COND_1719 & $\begin{array}{l}\text { https://bdoh.irstea.fr/DRAIX/GALABRE/COND } \\
\end{array}$ & $17-19$ \\
\hline & & $\begin{array}{l}\text { Major ion } \\
\text { concentrations }\end{array}$ & hitps://dolo.org/10.17178//DRAXXBLEOEN_GAL_ROB_ION_1719 & & $17-19$ \\
\hline
\end{tabular}

188 Table 1: Summary of the data set of the Galabre watershed of the Draix-Bleone observatory. 